\title{
Detecting and Compensating Sensor Faults in a Hydraulic Condition Monitoring System
}

\author{
Nikolai Helwig $^{1,2}$, Eliseo Pignanelli, ${ }^{1,2}$, Andreas Schütze ${ }^{2}$ \\ ${ }^{1}$ ZeMA - Centre for Mechatronics and Automation gGmbH, Saarbrücken, Germany \\ ${ }^{2}$ Lab for Measurement Technology, Saarland University, Saarbrücken, Germany, \\ Email: n.helwig@mechatronikzentrum.de
}

\begin{abstract}
In this work, we present a statistical condition monitoring system allowing the qualitative and quantitative determination of typical faults related to the hydraulic system as well as sensor faults of the installed sensor network. Single sensor faults such as constant offset, drift, noise, and signal peaks can be identified before leading to false alarms. Furthermore, the system is able to compensate the failure of up to 5 sensors with sufficient classification performance exploiting inherent correlations of sensor signals. Feature extraction, selection, and classification using LDA are fully automated. Thus, the system can react to a detected sensor fault and adapt the sensor data used for analysis significantly improving the robustness of condition monitoring system.
\end{abstract}

Key words: condition monitoring, fault detection, hydraulic system, sensor fault.

\section{Introduction}

Since recent decades, condition monitoring of industrial and mobile hydraulic applications, as a significant part of the condition-based maintenance strategy, is growing in importance [1]. Thereby, machine downtimes and maintenance costs can be reduced, but also planning security of production processes can be enhanced considerably. However, not only the machine to be monitored but also the condition monitoring system (CMS) itself can be affected by failures: A small undiscovered sensor malfunction, such as signal degradation caused by drift or noise, can result in gradual misclassification of the monitored system state. In this case, it is difficult to distinguish between the state of the monitored component and the underlying sensor fault. In many industrial applications, sensors and electronics are faced with harsh environments like a wide range of operating temperatures, strong electromagnetic fields, and aggressive media, which can affect the sensor signals. Also wiring problems, sensor cross-sensitivities and ageing of sensor elements are challenges for the robustness and reliability of CMS. There are different publications addressing sensor fault detection using symbolic dynamic filtering [2], Fuzzy voting and Kalman filtering [3], distributed filtering [4], and uncertainty-based distribution analysis [5]. Zug et al. used failure mode and effects analysis (FMEA) to identify and exclude faulty sensor signals in a sensor network [6]. Furthermore Tang et al. proposed an approach for the trustworthiness analysis of alarms based on sensor data in cyber-physical systems [7]. In this work, we expand the previously proposed automated statistical method for fault detection in hydraulic systems [8,9] to the detection of typical sensor faults such as constant offset, drift, noise and sudden signal peaks.

\section{Concept and experimental system}

Fig. 1 illustrates the procedure to train the CMS: Training data is collected via a modified hydraulic test bench which simulates different component conditions of cooler C1 (cooling efficiency degradation), valve V10 (impeded spool), main pump MP1 (internal leakage) and accumulator pre-charge pressure loss, here simulated with four accumulators A1 - A4 at different pre-charge pressures. The test bench is equipped with process sensors measuring pressures (PS1-6), volume flows (FS1-2), electrical pump input power (EPS1), temperatures (TS1-4) and vibration (VS1), furthermore three virtual sensors for cooling efficiency, cooling power and system efficiency are derived from physical sensor values. In total, 17 sensor signals are stored while the hydraulic system repeats pre-defined constant working cycles with changing conditions of hydraulic components to identify typical signal patterns. The working cycle (duration $60 \mathrm{sec}$ ) consists of different segments with transient 
and static load characteristics performed by the proportional pressure relief valve $\mathrm{V} 11$ and the directional valve V10 simulating a typical machine operation. After collecting raw sensor data during characterization measurement, the data for each cycle are split into 13 segments (valve V10 operation, static and transient loads) extracting features such as statistical moments (median, variance, skewness, and kurtosis) and signal shape parameters (fit of slope, position of maximum value) in each segment. From the resulting pool of 1326 features the 20 most significant features were chosen using the Pearson correlation with the four simulated hydraulic faults as selection criterion (feature selection). The final step is the dimensional reduction of the feature vector using LDA, here from 20 to 2 dimensions (discriminant functions, DF), and subsequent classification using the knearest-neighbor algorithm.

Furthermore, the raw signals of relevant sensors used for hydraulic fault monitoring were modified by implementing typical sensor faults such as constant offset, drift, noise and signal peaks pointing to an incorrect function of sensor or electronics. Here, each sensor fault type and grade is mathematically superimposed on the complete characterization measurement. Thus, the robustness of CMS to sensor malfunctions is studied, but also the ability to detect these malfunctions using suitable sensor interdependency features, e.g. ratios and correlations.
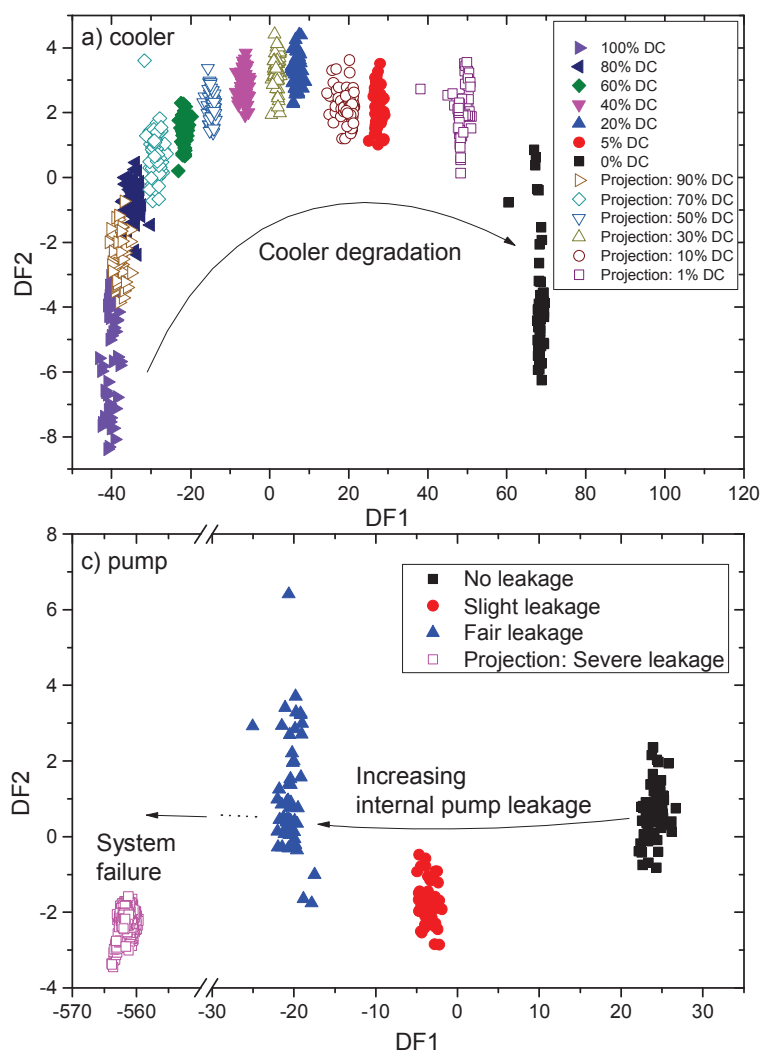

Fig 2. LDA space of typical hydraulic component faults (a) cooler degradation, (b) valve operation deterioration,

(c) internal pump leakage, and (d) gas leakage of accumulator using 20 features with trained and projected fault states.
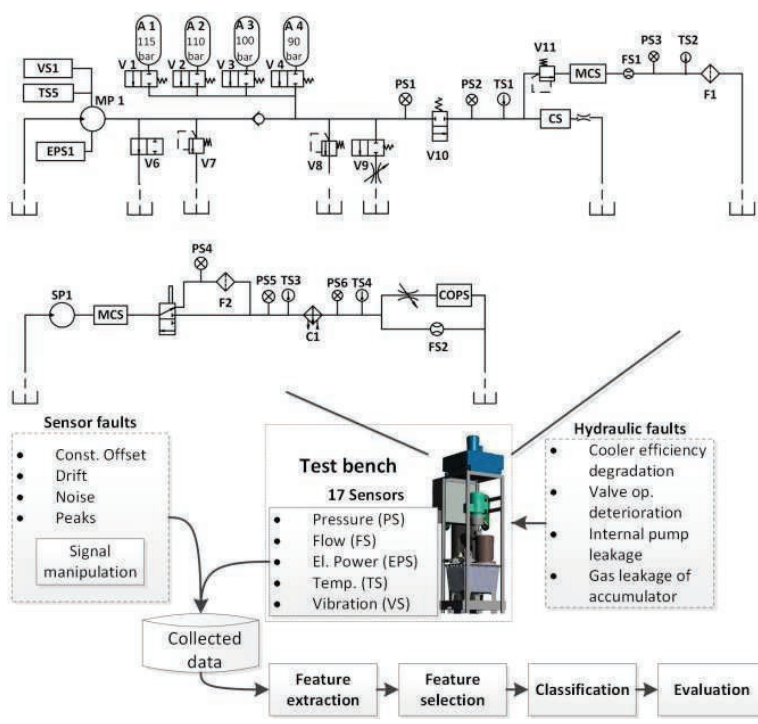

Fig.1. Hydraulic system and concept of data analysis

\section{Results}

As shown in fig. 2, fault-specific characteristic feature patterns can be obtained allowing the quantitative determination of fault severity grades in the hydraulic system in a twodimensional LDA space. Here, features from several fault grades are used for calculating the LDA coefficients ("training") while data from the further grades were projected to validate the ability to interpolate states that were not explicitly considered in training and verify the performance of statistical model. Features selected from correlation criterion for cooler
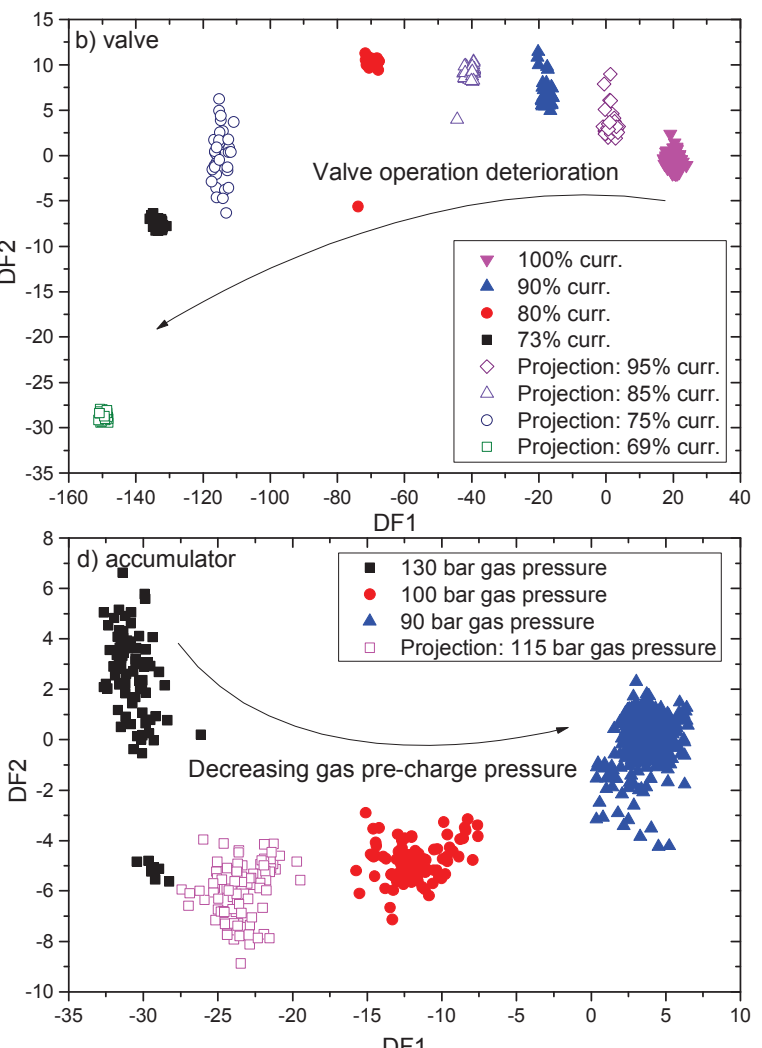
monitoring are median values of virtual sensors cooling efficiency and cooling power detecting an efficiency reduction of less than $1 \%$ simulated by a duty-cycle (DC) of $90 \%$ of the fan installed at cooler leading to a long-term fluid temperature increase of $0.5^{\circ} \mathrm{C}$. A dutycycle of $0 \%$ corresponds to a relative decline of $73 \%$ cooling power which results in oil temperatures higher than $80^{\circ} \mathrm{C}$. The monitoring of directional valve is based on pressure sensors installed before and after the valve analyzing the transient phase during valve operation using distribution (e.g. variance, skewness, and kurtosis) and signal shape (slope) functions. Here, the movement of spool is impeded by reducing the control current simulating an increased friction e.g. caused by substantial particle contamination of fluid. Even slight variations of current $(<2 \%)$ can be detected. Another analyzed fault type is internal pump leakage imitated by orifices bypassing high and low pressure ports of the pump. The leakage levels are $3.3 \%$ (slight), $4.6 \%$ (fair) of nominal pump flow rate. Furthermore, measurements of a hydraulic "short-circuiting" of pump were projected (severe leakage) by directly connecting the high pressure port to the tank. The features used for pump monitoring are median values of flow, system efficiency (virtual sensor), and pressures of static load phases. Especially piston-type accumulators are affected by gas leakage resulting in a
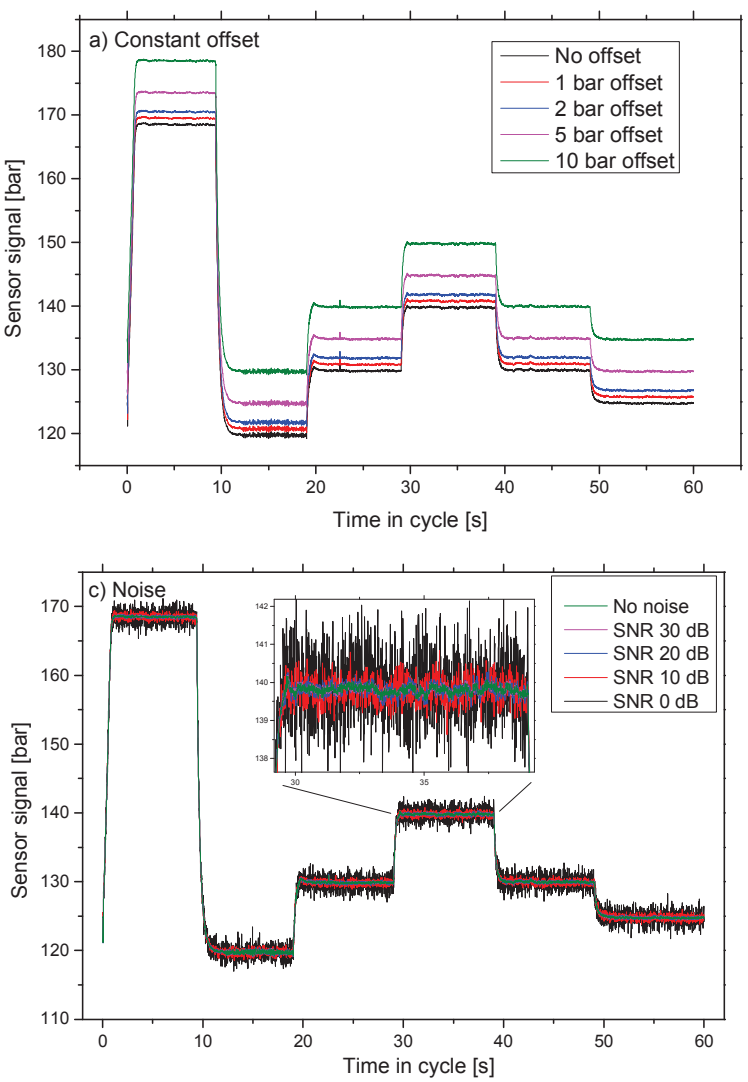

decrease of pre-charge pressure and a failure of operation in the long-term. The different precharge pressure levels of accumulator (fig. 2d) can be quantified by DF1, correctly projecting the intermediate state (115 bar) in LDA space. The significant features for accumulator monitoring are characteristics such as slope, variance, skewness, and kurtosis of transient load phases of power, pressure, and flow sensors.

In a further measurement, a constant working cycle with combinations of previously shown fault types including three cooling efficiencies $(100 \%, 20 \%$, and $3 \%$ fan duty cycle), three valve states $(100 \%, 85 \%$, and $73 \%$ control current), three pump leakage levels (inactive, slight and fair leakage) and four accumulator pre-charge pressures $(130,115,100$, and 90 bar) was analyzed within a fluid temperature interval from approx. $45^{\circ} \mathrm{C}$ to $65^{\circ} \mathrm{C}$. In this measurement sensor faults are superimposed: In case of "constant offset", four fault grades of pressure sensor PS1 are studied $(1,2,5$, 10 bar) as exemplarily shown in fig. 3a. Fig 3b illustrates the "baseline drift" of PS1 with different drift rates ranging from 0.5 to $5.0 \%$ per hour affecting the mean cycle value simulating a cross-sensitivity such as temperature change. Note that there is an inherent long-term change of the mean pressure in the measurement itself (especially
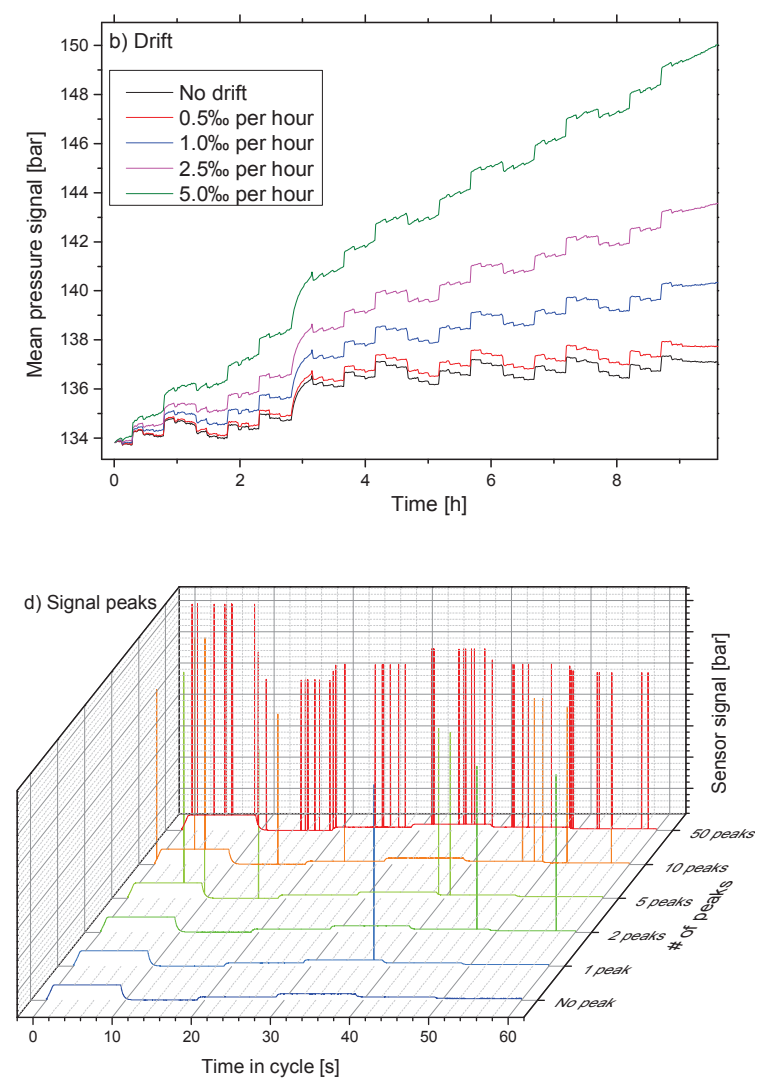

Fig. 3. Pressure sensor signals over operating cycle injecting the sensor faults (a) constant offset, (b) baseline drift, (c) noise, and (d) peak superposition with different grades of severity. 
around hour 3) which makes it difficult to differentiate between the different drift rates with an isolated sensor analysis. Furthermore, fig. 3c shows an artificially deteriorated sensor signal using Gaussian white noise with different amplitudes generating $30 \mathrm{~dB}, 20 \mathrm{~dB}, 10 \mathrm{~dB}$ and $0 \mathrm{~dB}$ signal-to-noise ratios (based on a normalized signal). Another sensor fault studied is the appearance of sharp short signal peaks with large amplitudes (fig. 3d) which do not reflect realistic measurements. Here the number of peaks which are randomly placed in the working cycle ranges from 1 to 50 .

Now the performance of CMS for hydraulic components is tested while the signals of most significant sensors are affected by drift and noise faults with different grades of severity (fig. 4). In general, there are considerable variations in the decline of detection rate dependent on hydraulic component. While the cooler monitoring is not affected at all by these sensors faults (constant $100 \%$ correct classifications), the valve monitoring detection rate especially decreases in presence of high noise amplitudes due to the use of susceptible transient features (fig. 4b). Compared to cooler and valve condition monitoring, pump and accumulator are highly prone to sensor disturbances. The pump leakage detection (fig. 4c) particularly degrades with increasing drift rates which cause significant shifts of the used median features. In case of accumulator (fig. 4d) only a slow drift rate of $0.5 \%$ per hour induces a sharp decline of detection rate from $95 \%$ to $42 \%$, also barely noticeable noise amplitudes (30 dB SNR) degrade the classification by more than $20 \%$. The validity of sensor signals consequently is an issue of importance which has to be considered in the condition monitoring system ensuring a robust and reliable analysis.

In the following analysis, sensor faults are applied to the single pressure sensor PS1 and classification target values are the grades of severity using the same measurement dataset as in fig. 4. The automated feature extraction and selection method corresponds to the hydraulic component monitoring (fig. 2), though the feature domains are different. In case of offset and drift disturbances, we use a cascaded feature extraction first computing median ratio values over the whole cycle of different sensors in the hydraulic system and subsequently analyzing the time-series of
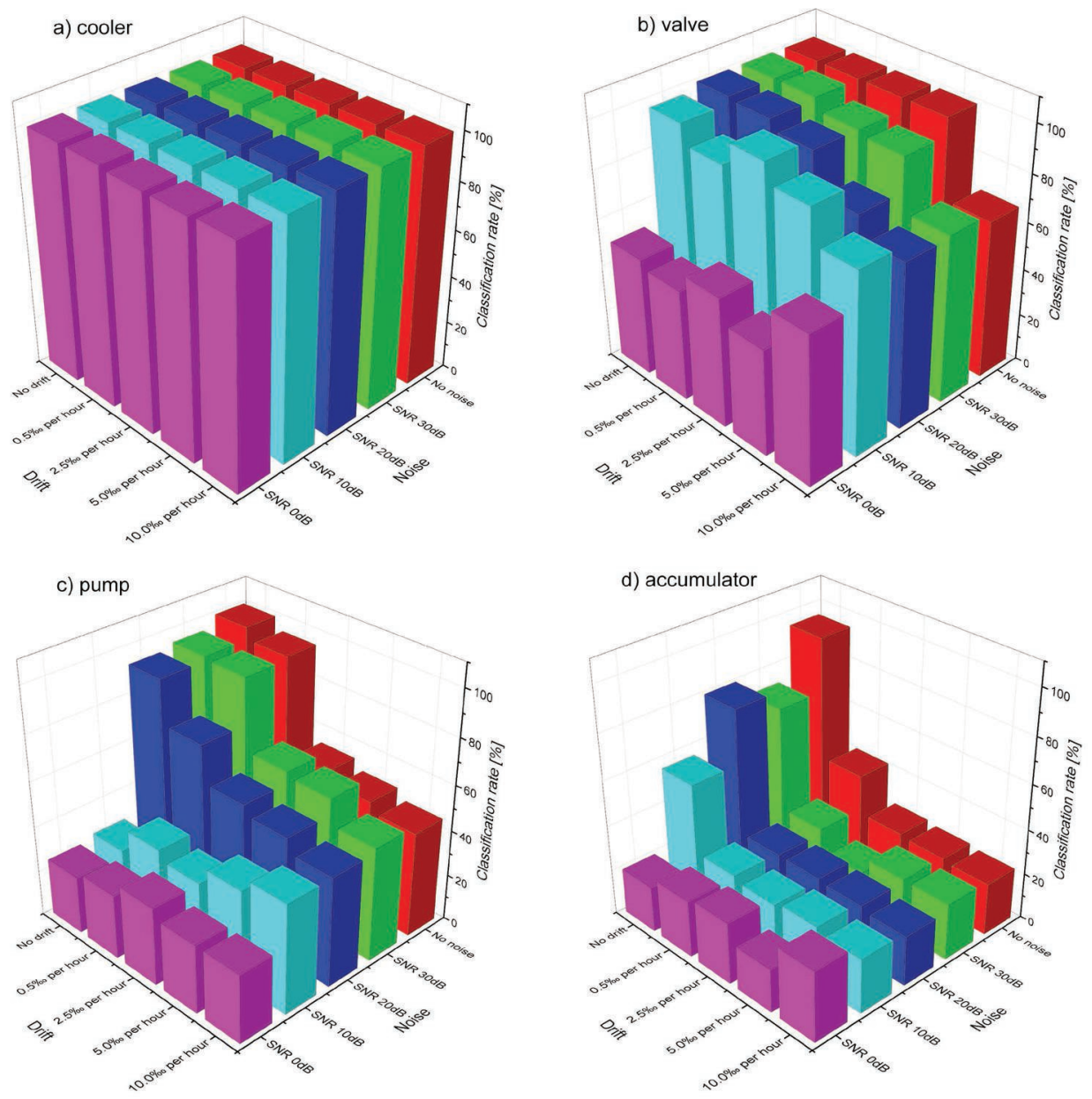

Fig. 4. Influence of sensor faults noise and drift affecting the relevant sensors on hydraulic fault detection performance for cooler (a), valve (b), pump (c), and accumulator (d) monitoring. 
previously obtained ratios within a window of 60 minutes. For noise detection, features are extracted from $17 \times 17$ cyclewise correlation matrix of sensor net with 136 independent pairwise linear correlation coefficients which are also used for time-series feature extraction (60 min). The peak detection is based on signal shape and statistical moments similar to the feature domain of hydraulic fault detection. Fig. 5 shows the LDA spaces of different sensor disturbance types using several fault grades for training and the other grades for evaluation. The constant offset (fig. 5a) can be detected for slight offset values in the range of 1 bar (which corresponds to an error of $0.4 \%$ of full scale) even though there is no clear class separation due to inherent signal ratio variations, and intermediate fault grades (2 bar, 10 bar) are properly projected. The drift detection (fig. 5b) exhibits a good class separation discriminating very slow drift rates (0.5\%o per hour) and normal sensor operation in typical application sensor data. Just as well, the statistical model for drift recognition is able to correctly interpolate fault grades not considered in training (1.5\% and $5.0 \%$ per hour). In case of noise monitoring (fig. 5c), the detection limit based on correlation features is $20 \mathrm{~dB}$ signal-tonoise ratio which is a good result exempt from accumulator monitoring where $30 \mathrm{~dB}$ SNR degrades noticeably the classification performance. Not only noise sensor faults can be identified but also sensor wiring problems such as cable break simulated by freezing the sensor value to zero with additional noise which results in DF1 values far in the negative range. Also the number of induced signal peaks (fig. $5 d$ ) is correctly quantified and the trained LDA can be successfully applied to test data. In summary, all studied sensor fault types can be identified with the presented approach and feature domains allowing and fault grade estimation with DF1 values. Here, only the presence of a single sensor disturbance was analyzed, the determination of several faulty sensors occurring simultaneously is aim of future work. Based on the shown sensor fault classification (fig. 5) and the susceptibility of CMS to sensor faults (fig. 4), limit values for DF1 pointing to sensor malfunction can be defined to decide whether the sensor data is invalid and should be ignored in actual diagnosis. In this case, the training data set has to be adapted by excluding the data of the concerned sensor and performing a re-training of feature selection and LDA to find the next best features for the specific component monitoring and compensate the sensor fault. In fig. 6, the ability of sensor network to compensate failures of most significant sensors is studied: Due to partially redundant sensor signals, the failure of up to 5 significant sensors can be compensated with acceptable classification rates (cooler, valve, pump) in the range of $90 \%$. Comparison between the two supervised classification methods LDA and
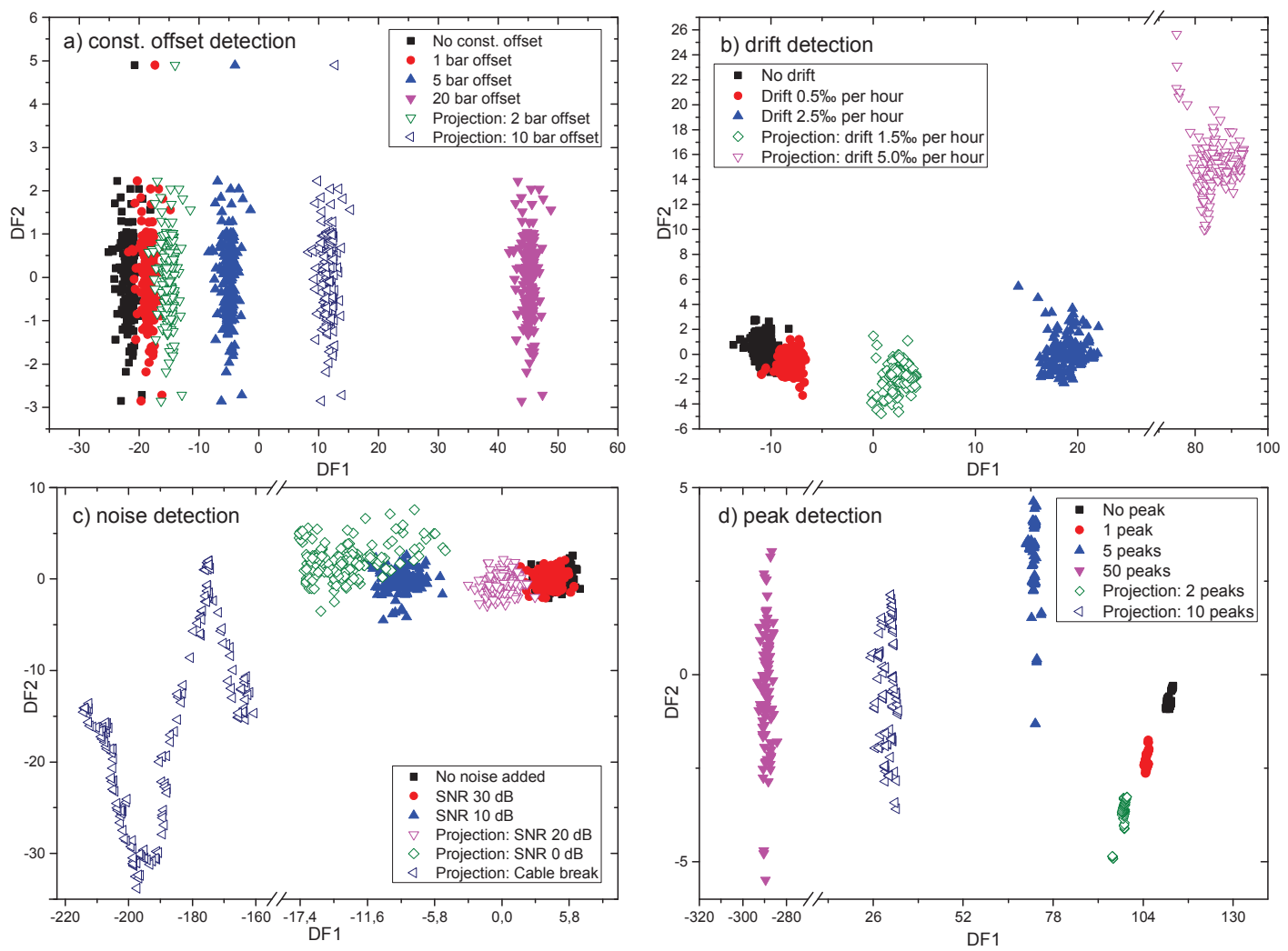

Fig. 5. LDA plots for sensor fault monitoring of (a) constant offset, (b) baseline drift, (c) noise, and (d) peak superposition each with trained and proiected states for validation. 
artificial neural network (ANN, multilayer perceptron with 10 hidden layers) shows slightly higher detection rates (up to $20 \%$ ) for ANN, especially for challenging classification data with many excluded sensors.

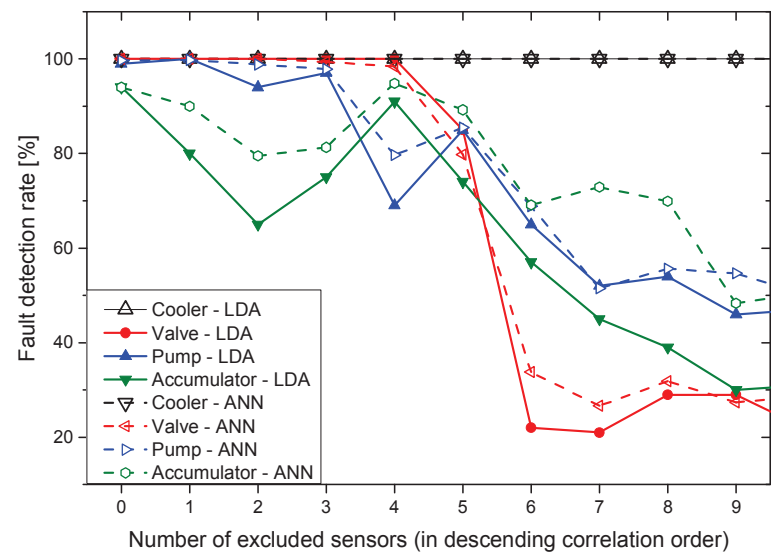

Fig. 6. Classification rate of hydraulic system fault vs. number of excluded sensors for LDA with k-nearestneighbor $(k=3)$ classifier and for a multilayer perceptron with 10 hidden layers.

\section{Conclusion}

The presented condition monitoring approach covers a wide range of detectable fault types such as faults related to the hydraulic system as well as different kinds of sensor malfunctions. Automated feature extraction, selection and supervised learning were proven to be suitable for the identification and severity grade estimation of typical sensor faults such as constant offset (detection limit $0.4 \%$ error FS), drift (detection limit $0.5 \%$ per hour), noise (detection limit $20 \mathrm{~dB}$ SNR) and signal peaks (single peak detection limit). Therefore, timeseries interrelation features of the sensors in network are used like median ratios and pairwise correlations. In case of a sensor fault, the affected sensor can be identified automatically and substituted by the sensor network considerably improving the overall reliability of the CM system. By exploiting hidden signal redundancies using correlation criteria, it has been demonstrated that the system compensates the failure of up to 5 sensors including significant information with marginal degradation of classification performance.

\section{Acknowledgement}

This project was funded through the EFI program (support of development, research, and innovation in Saarland) and financed by HYDAC Filter Systems $\mathrm{GmbH}$ (Sulzbach, Germany).

\section{References}

[1] Philip Higgs et al., "A Survey on Condition Monitoring Systems in Industry," ASME 7th Biennial Conference on Engineering Systems Design and Analysis, No. ESDA2004-58216, pp. 163-178, 2004.

[2] Soumik Sarkar, Jin Xin, and Ray Asok, "Data-driven fault detection in aircraft engines with noisy sensor measurements." Journal of Engineering for Gas Turbines and Power 133.8, 2011.

[3] Sebastian Blank, Thomas Pfister, and Karsten Berns, "Sensor failure detection capabilities in low-level fusion: a comparison between fuzzy voting and Kalman filtering." Robotics and Automation (ICRA), 2011 IEEE International Conference on. IEEE, 2011.

[4] Hongli Dong et al., "A Survey on Distributed Filtering and Fault Detection for Sensor Networks." Mathematical Problems in Engineering, 2014.

[5] Yang Yang et al., "An Uncertainty-Based Distributed Fault Detection Mechanism for Wireless Sensor Networks." Sensors 14.5, 2014.

[6] Sebastian Zug, André Dietrich, and Jörg Kaiser, "Fault-Handling in Networked Sensor Systems." Fault Diagnosis in Robotic and Industrial Systems, 2012.

[7] Lu-An Tang et al., "Trustworthiness analysis of sensor data in cyber-physical systems." Journal of Computer and System Sciences 79.3, 2013.

[8] Nikolai Helwig, Eliseo Pignanelli, and Andreas Schütze, "Condition Monitoring of a Complex Hydraulic System using Multivariate Statistics." 2015 IEEE International Instrumentation and Measurement Technology Conference, in press, 2015.

[9] Nikolai Helwig, and Andreas Schütze, „Intelligentes Condition Monitoring mit automatisierter Merkmalsgenerierung und -bewertung"; in: A. Schütze, B. Schmitt (Hrsg.): XXVIII. Messtechnisches Symposium des Arbeitskreises der Hochschullehrer für Messtechnik, Tagungsband, Shaker Verlag, Aachen (2014), ISBN 978-3-8440-2994-9, S. 121128. 